development of the expression for the density of the prime ideals of a class, and its application to the problem of the determination of the number of classes. This is followed by a consideration of the zeta function and some of its applications.

Chapter seven is devoted to a more detailed consideration of quadratic fields. Norm residues are considered and the properties of Hilbert's norm residue symbol are developed. The separation of the ideals into genera is studied and the number of genera determined.

The further topics considered in this chapter are the zeta function in a quadratic field; the expression for the class number in a quadratic field (this without the use of the zeta function); the Gaussian sum; and the relations existing between ideals of a quadratic number field and binary quadratic forms.

Chapter eight contains a new proof of the most general law of quadratic reciprocity in an arbitrary algebraic number field. In this development the author makes use of his generalization of the Gaussian sum.

The chapter ends with a proof of the existence of class fields of relative degree two. This is a consequence of the general law of reciprocity.

\title{
G. E. WAHLIN
}

Die Philosophischen Grundlagen der Wahrscheinlichkeitsrechnung. By

E. Czuber. No. XXIV of Wissenschaft und Hypothese. Leipzig and Berlin, B. G. Teubner, 1923. $8+343$ pp.

Czuber's book on the philosophy of probability is in essence a clarification of the fundamental concepts and theorems of the theory of probability by means of analysis and criticism of attacks directed against the theory in certain recent writings. The author's patient analysis and kindly criticism center chiefly on Meinong's rather labored Über Möglichkeit und Wahrscheinlichkeit and Marbe's naive ideas on probability expressed in his Die Gleichförmigkeit in der Welt. The concept and definition of probability, the addition and multiplication theorems, the theorems of Bernonlli, of Poisson, and of Bayes, the relation of probability to our knowledge of nature, all come in for detailed discussion.

Czuber's book, in the opinion of the reviewer, is intended primarily for non-mathematicians. Students of mathematical probability will find in the author's admirable Wahrseheinlichkeitsrechnung (3d edition, 1914) the substance of nearly all his views on the fundamentals of probability found in the Grundlagen. One instance of departure from his earlier work may be worth noting here: the obtaining (in Chap. VI) of the theorem of Bayes as a simple corollary of the multiplication theorem for dependent events (compare Boole's Laws of Thought, p. 254, III).

\section{B. A. Bernstein}

